\title{
James A. Beckford, Social theory and religion
}

Cambridge, Cambridge University Press, 2003, 251 p. (bibliogr., index, tablx.)

Anne-Sophie Lamine

\section{(2) OpenEdition}

\section{Journals}

Édition électronique

URL : http://journals.openedition.org/assr/1930

DOI : $10.4000 /$ assr. 1930

ISSN : $1777-5825$

Éditeur

Éditions de l'EHESS

\section{Édition imprimée}

Date de publication : 1 octobre 2004

Pagination : $53-158$

ISBN : 2-222-96754-6

ISSN : 0335-5985

Référence électronique

Anne-Sophie Lamine, « James A. Beckford, Social theory and religion », Archives de sciences sociales des religions [En ligne], 128 | octobre - décembre 2004, document 128.5, mis en ligne le 16 novembre 2005, consulté le 24 septembre 2020. URL : http://journals.openedition.org/assr/1930 ; DOI : https://doi.org/ 10.4000/assr. 1930 
Globalement, la vie sociale des aumôneries inclut assez peu les ministres-visiteurs et la prise en compte des sensibilités culturelles non-chrétiennes est très variable selon les aumôniers et selon les ressources disponibles. L'analyse de ces relations montre bien que les groupes religieux «n'interagissent pas dans le vide », mais dans un contexte spécifique de concurrence, avec des enjeux de reconnaissance et de ressources matérielles et symboliques à partager et dans un contexte institutionnel contrôlé par les autorités carcérales.

Une comparaison avec le système d'aumôneries de prisons américaines apporte un éclairage complémentaire à l'analyse (septième chapitre). À la différence de la Grande-Bretagne, la diversité religieuse des États-Unis est surtout intrachrétienne, puisque seuls $3 \%$ des Américains se déclarent d'une religion non-chrétienne et il y a séparation formelle entre Églises et État. Alors que la situation anglaise est qualifiée par les AA. de "relativement opaque ", "dépendant de situations inégalitaires de patronage » et fonctionnant de manière « ad hoc », la situation américaine est plus systématisée, plus égalitaire, plus « transparente » et moins flexible (p. 171). Par exemple, face à la demande de régimes alimentaires particuliers, les prisons fédérales offrent le choix de s'inscrire au « régime spécial commun » sensé être équitable et convenir plus ou moins à tous les groupes : viande casher (donc acceptable pour les musulmans), vaisselle ne servant que pour ce régime.

Il ne faudrait pas déduire au terme de cette étude que l'Église établie utilise ses privilèges pour écarter les « autres religions » de l'aumônerie. Les observations des AA. montrent que ses relations avec les «autres religions » sont bien plus complexes. En particulier, l'implication personnelle et la bonne volonté de nombreux aumôniers apparaissent clairement. Ceux-ci n'en reflètent pas moins le caractère établi de l'Église d'Angleterre, en jouant des rôles d'intermédiaires et de facilitateurs entre les ministres et l'administration. Finalement, l'aumônerie de prison s'avère un excellent analyseur de la prise en compte de la pluralité religieuse dans la société plus large. Comme le concluent les AA., si l'Église d'Angleterre a pu apparaître comme le dais qui protège les « autres religions », il semble que ce dais soit maintenant percé de «larges trous » (p. 203). Cette étude a aussi l'immense mérite de montrer la nécessité de préciser la notion de pluralisme. Car si le système analysé semble à première vue pluraliste, l'analyse plus fine des rapports entre groupes religieux et entre ces groupes et l'administration pénitentiaire dévoile une distribution des ressources et de la reconnaissance qui est loin d'être égalitaire. En outre, alors que la diversité religieuse a souvent été considérée comme relevant de la sphère privée, le cas des prisons montre bien que cette pluralité relève aussi des politiques publiques. L'ouvrage apporte à la fois un très riche matériau empirique et des analyses stimulantes sur la gestion concrète de la pluralité religieuse dans la société et dans ses institutions ainsi que sur l'évolution des relations entre État et religions.

Anne-Sophie Lamine.

BECKFORD (James A.).

Social Theory and Religion. Cambridge, Cambridge University Press, 2003, 251 p. (bibliogr., index, tablx.).

J.A.B. part du constat que très peu d'études combinent la sociologie des religions et la théorie sociologique. Son objectif est alors de montrer qu'une meilleure connaissance mutuelle serait très féconde pour l'une comme pour l'autre. Il appuie sa démonstration sur quatre dossiers : la sécularisation, le pluralisme, la globalisation et les nouveaux mouvements religieux. Il se place dans le cadre d'une approche constructiviste, dont il présente les atouts pour aborder le champ religieux tout en soulignant qu'elle est complémentaire d'autres méthodes d'analyse ( $1^{\text {er }}$ chapitre). Il s'agit d'accorder la priorité à l'analyse des processus impliqués dans la négociation des significations du phénomène social étudié. Il s'agit avant tout de savoir comment des individus, des groupes, des organisations utilisent, justifient et institutionnalisent l'usage de termes comme religieux, sacré, spirituel et régulent les activités correspondantes. Cela est particulièrement pertinent pour approcher les frontières changeantes du religieux : la limite public-privé, le religieux acceptable ou non etc.

Dans les quatre chapitres suivants, l'auteur confronte une série de théories sociologiques avec les résultats d'études de sociologie des religions. Il montre d'une part, certaines insuffisances de ces théories, lorsqu'elles approchent le fait religieux et d'autre part, l'intérêt que certains concepts théoriques auraient à être transposés à l'étude du champ religieux.

Le premier dossier théorique abordé par l'A. est celui de la sécularisation. Il souligne d'abord que l'approche des caractéristiques sociales de la religion implique le plus souvent l'hypothèse de son déclin, ce qui la différencie de l'étude d'autres domaines comme l'éducation ou la santé. J.A.B. décline six filières théoriques de la formation du concept de sécularisation : la 
différenciation structurelle, l'empirisme (et les idées scientifiques), la dynamique interne de la religion (surtout le christianisme), les visions libérales (politiques et sociales), le marxisme et le freudisme. La notion de sécularisation n'a donc jamais eu une signification unique. De plus elle s'est formée autour de disputes philosophiques et idéologiques. L'A. propose ensuite une carte des approches actuelles de la sécularisation en synthétisant les apports de leurs principaux auteurs, en six sections : la différenciation, la rationalisation, la modernisation, les métamorphoses, la persistance de la vitalité et l'universalité de la religion. Il discute chacune de ces approches et ses hypothèses implicites, et propose des pistes de recherche. Ainsi, à propos de la rationalisation, il observe que « la force argumentative des effets sécularisants de la rationalisation dépend fortement de l'hypothèse que les manières religieuses et rationnelles de penser sont mutuellement exclusives. » (p. 48). À propos des métamorphoses, il suggère d' " examiner la reconceptualisation de ce qui compte comme religion dans la vie publique et dans la vie privée » (p. 54) et même les "procédés par lesquels on construit une définition du privé et du public». J.A.B. conclut en disant que c'est bien parce qu'on est très peu conscient du fait que la religion est le produit d'une construction sociale que les débats sur la sécularisation s'apparentent souvent à des " dialogues de sourds ». Il pousse donc à s'intéresser davantage aux changements de signification attribués à la sécularisation en divers temps et en divers lieux et à accorder plus d'attention aux processus sociaux de construction et de négociation de la frontière entre le religieux et le séculier.

Le second dossier théorique est celui du pluralisme. Le chapitre intitulé « les caprices du pluralisme », a d'abord l'immense mérite de proposer une clarification du concept. L'emploi du terme «pluralisme» par les sociologues correspond en effet à trois aspects distincts de la place de la religion dans la société : la diversité religieuse, l'acceptation de la diversité religieuse et le pluralisme comme acceptation positive de la diversité. La diversité religieuse peut concerner le nombre d'organisations religieuses (en tenant compte ou non de leur taille), le nombre de religions distinctes, la pluralité des ressources religieuses auxquelles les individus peuvent puiser ou encore la diversité interne des courants religieux. Le second aspect est celui de la reconnaissance et de l'acceptation des groupes religieux. Il peut inclure les dispositions légales et constitutionnelles, le fait qu'un groupe religieux soit le point de référence (implicite ou explicite) en matière de religion, mais aussi l'acceptation que les groupes religieux ont des autres groupes. Enfin, le pluralisme comme acceptation positive de la diversité correspond au pluralisme comme valeur. Il peut signifier que la diversité des groupes est une bonne chose pour la société, ou qu'elle est bonne pour l'individu, ou encore qu'il est bon que les individus combinent des éléments des différentes traditions. À cela, il faut ajouter que le pluralisme n'apparaît pas dans le vide, mais qu'il est débattu, contesté ou affirmé dans divers contextes sociaux et politiques. J.A.B. propose ensuite une revue de l'utilisation de ce terme par des sociologues de la religion. Il donne une place importante à l'approche de P. L. Berger, à qui il adresse deux critiques. D'une part, ce dernier exagère l'effet de la fin du caractère obligatoire de la religion et celui la diversification de la scène religieuse sur la fragilisation des croyances et sur leur crise de crédibilité. D'autre part, son approche élude les difficultés liées à la distinction privé-public. J.A.B. fait ensuite référence aux quelques sociologues qui ont abordé la gestion publique de la diversité religieuse, pour souligner l'importance du contexte politique. Il utilise aussi l'exemple des aumôneries de prison anglaises (voir la recension de l'ouvrage qu'il a écrit avec S. Gilliat sur ce sujet, Religion in Prison (cf. Arch. supra 128.4), pour montrer qu'une situation apparemment pluraliste révèle des inégalités réelles dans la distribution des ressources et de la reconnaissance.

Le troisième dossier théorique est celui de la globalisation. Fidèle à son approche constructiviste, l'A. rappelle que la globalisation est aussi une construction sociale et qu'on ne peut pas simplement chercher à étudier la réponse sociale des divers groupes à cette globalisation. Il souligne surtout que très peu d'études sur la globalisation abordent le champ religieux, ou qu'elles le font brièvement et sur un aspect très spécifique. Pourtant, la religion pose de nombreux défis à la globalisation. D'abord « la religion est par définition réflexive », ensuite, elle est extrêmement diversifiée, y compris dans ses approches et ses réponses à la globalisation. J.A.B. passe en revue quatre auteurs ayant contribué à la conceptualisation de la globalisation : Beck, Waters, Scholte, Giddens. Il regrette globalement que ces études fassent très peu cas des recherches empiriques de sociologie des religions et réduisent le plus souvent la religion au fondamentalisme. Or, les phénomènes religieux actuels sont loin de se limiter à cette dimension, qu'il s'agisse de la religion ordinaire ou populaire, des tendances libérales, du Nouvel Âge, du développement des relations entre groupes religieux ou encore des divers types de régulation du religieux par les États. J.A.B. insiste cependant sur l'intérêt qu'il y aurait à 
utiliser des notions développées par ces théoriciens, tout en les amendant de manière adéquate, pour étudier le champ religieux.

Le quatrième dossier théorique porte sur les nouveaux mouvements religieux. J.A.B. observe là encore que les théoriciens des mouvements sociaux, de la cognition, de la culture et de l'identité ont très largement ignoré les mouvements religieux dans leurs analyses. L'influence des approches marxistes a contribué à écarter toute possibilité de forme libératrice ou résistante du religieux. Réciproquement, très peu d'études sur les nouveaux mouvements religieux utilisent les concepts développés par les sociologues des mouvements sociaux (conflits de classe, dissonance cognitive ou anomie...). Il souligne le bénéfice qui pourrait être tiré de la théorie du choix rationnel, tout en signalant les inconvénients des approches trop restrictives de la rationalité qui est d'ailleurs « trop importante pour être laissée aux théoriciens du choix rationnel » (p. 169). L'A. souligne les « implications mutuelles entre ces idées théoriques et l'interprétation scientifique des mouvements religieux », qui incluent l'effort des mouvements religieux pour s'approprier un espace identitaire, les controverses sur les «sectes» dans un contexte d'incertitudes sociales et culturelles et les relations entre identités réflexives et postmodernité. L'étude des mouvements religieux pourrait aussi offrir une bonne manière d'explorer la « géographie énigmatique et changeante des frontières entre public et privé » ainsi que la «bataille entre les notions d'individualisation et de collectivisation ».

Voilà donc un ouvrage extrêmement stimulant à la fois pour sa richesse bibliographique impressionnante, son esprit de synthèse et d'analyse, sa mise en perspective de recherches qui s'ignorent et la quantité de suggestions constructives pour des travaux futurs. On pourrait certes souhaiter y trouver un peu plus de références d'études portant sur les religions nonchrétiennes. Mais il s'agit bien là d'un ouvrage qui va devenir une référence dans la discipline. En plus d'y trouver une synthèse remarquable sur quatre grandes questions : la sécularisation, la pluralité, la globalisation et les nouveaux mouvements religieux, les sociologues de la religion y liront de nombreuses voies de recherche prometteuses, les invitant à rapprocher leur matériau aux réflexions sociologiques théoriques, à en utiliser certains concepts et à en critiquer ou amender certaines conclusions. Il sera extrêmement utile aussi bien aux chercheurs débutants pour le point qu'il fait sur des questions centrales et pour les nombreuses pistes suggérées, qu'aux chercheurs confirmés qu'il met au défi de reconsidérer leur manière de définir la religion et ses frontières et de « sortir de leur isolement » en se confrontant davantage aux travaux de leurs collègues. Le défi lancé aux «théoriciens » de la modernité n'est pas moindre, l'A. leur montre qu'ils ne peuvent plus ignorer le champ de la religion ni le réduire à quelques idées simples comme le retour du réprimé ou le fondamentalisme.

Anne-Sophie Lamine.

BEREND (Nora).

\section{At the Gate of Christendom. Jews, Muslims} and 'Pagans' in Medieval Hungary, c.1000c.1300. Cambridge, Cambridge University Press, 2001, 340 p. (bibliogr., index, cartes, annexes) (coll. « Cambridge Studies in Medieval Life and Thought, Fourth Series »).

N.B. enseigne à Saint Catherine's College, University of Cambridge. Depuis septembre 2002, elle dirige le projet 'Christianization and State-formation in Northern and Central Europe, c. 900-c.1200' (Center for Research in the Arts, Social Sciences and Humanities, University of Cambridge http : IIwww.crassh. cam.ac.uk/ projects/christianization.html).

Le titre de l'ouvrage de N.B. rappelle qu'avant de devenir le «Bastion » de la chrétienté, le royaume hongrois, lui-même tout récemment converti, a tenu une position moins abruptement défensive à l'égard des nonchrétiens, qu'il fut tout à la fois une «porte » vers la chrétienté et n'en fut lui-même « qu'à la porte ». En introduction l'auteur présente ainsi son travail : " Ce livre compare le destin de trois groupes, les juifs, les musulmans et les coumans 'païens' dans la Hongrie médiévale ». Le but poursuivi est double. Dans un premier temps, présenter une étude de cas qui contribue à notre connaissance des populations non-chrétiennes vivant dans l'Europe médiévale, en intégrant l'Europe non-occidentale dans le champ d'analyse du monde médiéval. Dans un second temps, le but est d'examiner divers aspects de la place des minorités religieuses dans ce qui fut, « une société frontière. » (p. 2).

Après une annonce aussi riche et complexe, de façon très analytique, point à point, N.B. présente le cadre de son étude et analyse les communautés étudiées. Le chapitre 1 est consacré à la Hongrie en tant que société frontière. Ce thème semble au cœur des préoccupations de N.B. qui reprend tout d'abord la question du concept de société frontière puis l'usage fait par les médiévistes de ce concept. Comme elle le rappelle judicieusement (p. 17) des connaissances préalables de l'histoire de la 